\title{
Compact Micromachined Infrared Bandpass Filters for Planetary Spectroscopy
}

\author{
Willie C Merrell II, Shahid Aslam, Ari D Brown*, James A Chervenak, Wei-Chung Huang, \\ Manuel Quijada, Edward Wollack
}

NASA Goddard Space Flight Center, Greenbelt, MD 20771

* Corresponding author ari.d.brown@nasa.gov,

\section{Abstract}

The future needs of space based observational planetary and astronomy missions include low mass and small volume radiometric instruments that can operate in high radiation and low temperature environments. Here we focus on a central spectroscopic component, the bandpass filter. We model the bandpass response of the filters to target the wavelength of the resonance peaks at 20,40, and 60 $\mathrm{mm}$ and report good agreement between the modeled and measured response. We present a technique of using common micromachining processes for semiconductor fabrication to make compact, free standing resonant metal mesh filter arrays with silicon support frames. The process can accommodate multiple detector array architectures and the silicon frame provides lightweight mechanical support with low form factor. We also present a conceptual hybridization of the filters with a detector array.

OCIS codes: $040.2235,040.1240,120.2440,220.4000$

\section{Introduction}

A new generation of filter radiometers, gratings, and Fourier transform spectrometers are required for the investigation of the cold outer planets of the solar system and their icy moons [1]. The new instrumentation must operate in a cryogenic high radiation environment, be light-weight, and show high radiometric signal-to-noise performance. For instance, the thermal instrument straw-man payload of JEO on EJSM [2] for mapping out thermal anomalies, structure, and atmospheric compositions of Europa and Jupiter within the 7-100 $\mu \mathrm{m}$ spectral wavelength range calls for a mass budget of $3.7 \mathrm{~kg}$ and must operate under a total ionizing dose rate of $2.9 \mathrm{Mrad} /$ year [3]. Key optical components to such instruments are high transmission bandpass filters for the mid-far IR spectral region.

One scheme for achieving high peak transmission coupled with good out-of-band attenuation is an array of cross-shaped or annular apertures in thin metal foils of films [4]. Cross-shaped metal meshes and their complimentary inverse structures have, respectively, inductive (high pass) and capacitive (low pass) responses to incident light. Babinet's principle in vector formulation states that complementary meshes have complementary transmittance [5], i.e., a maximum for the inductive mesh is a minimum for the capacitive mesh. Thus, a mesh can be made that is both inductive and capacitive which results in a self resonant mesh with a bandpass response. The incident light excites a standing wave on the surface of the mesh, and transfers the incident energy into reflected and transmitted light. The inductive, capacitive, and resonant meshes are usually fabricated out of thin metal foils. These can consist of structures, either free 
standing or hot pressed into a dielectric media [6], in which the interlayer spacing, if present, is appropriately controlled. For spectral wavelengths $>20 \mu \mathrm{m}$ multilayer non-crystalline dielectric filters are not suitable for applications which need high in-band transmissivity and high out-of-band rejection, because the required layer thickness results in high absorption by the dielectric [7]. Furthermore, it is unclear whether or not ageing arising from exposure in high radiation environments will affect the mechanical strength and optical properties of the dielectric [8]. Also, in the high radiation environments encountered in spaceflight one needs to consider deep dielectric charging and spurious light generation due to high energy charged particles (e.g., cosmic rays), interacting with the dielectric volume. From this perspective all metal (or metal coated) structures are easier to deal with, than materials with low intrinsic conductivity, to integrate in future outer planet thermal instruments.

An excellent review of the development of metal mesh filters has been given by Goldsmith [9]. One early method proposed by Huggard et al [10] was the use of commercial precision printing screens, consisting of freestanding electroformed nickel sheets with a regular array of circular holes to fabricate large area band pass filters. The screens were robust and showed high peak transmittance between $70 \mu \mathrm{m}$ and $172 \mu \mathrm{m}$ in spectral wavelength. More recently, Melo et al [11] successfully fabricated suspended resonant nickel metal mesh filters for discrete central wavelengths ranging from $30 \mu \mathrm{m}$ to $750 \mu \mathrm{m}$ using standard photolithographic techniques and their measured transmission and bandwidths correlated well with the simulations from a 3D EM simulator.

In this paper, we report on the design, 3D EM simulation, and fabrication of FIR band pass filters constructed using microelectromechanical system (MEMS)-based fabrication techniques which result in high transmission at select wavelengths, namely $20 \mu \mathrm{m}, 40 \mu \mathrm{m}$, and $60 \mu \mathrm{m}$.

We demonstrate a technique of using common micromachining processes for semiconductor fabrication to make compact, free-standing resonant metal mesh bandpass filter arrays using silicon support structures. Silicon is lightweight, has low form factor and its mechanical support is robust in that it provides protection against deformation while handling and stacking the filters. The frame can also be used a spacer for filter stacking or extended to include baffles to prevent crosstalk between the detector channels. Using photolithography to make the frames also increases the fabrication throughput, because multiple frame architectures can be produced on a single wafer. Our primary discussion will center on the fabrication of the filters on a silicon frame and characterizing their spectral bandpass response. We choose copper as a radiation hard material whose electrical conductivity is greatly enhanced at low temperature which will result is reduced signal loss. As part of our design and characterization effort, we model the bandpass response of different filter element shapes to target peak transmittances at 20,40, and 60 micron wavelengths. We report good agreement with the modeled and measured bandpass response. We will also present a strategy for hybridizing the filter array with a sensor array for future integration into a mid - far IR spectrometer or radiometer design.

This paper is organized as follows. In Section I we discuss the modeling of the filter response. In Section II we describe the fabrication of the filters. In Section III we discuss the transmission measurement of the filters and compare the results with the model. We provide a future outlook in Section IV and conclude the paper in Section V.

\section{Section I: Filter Modeling}


We desired a modeling framework capable of predicting resonant metal mesh filter response prior to fabrication to help target specific passbands, for a review of the nature of the resonant metal mesh filter response see [6]. The starting point for the modeling approach employed here follows the work of Porterfield et al [12]. Specifically we used Ansys' 3D electromagnetic solver HFSS $^{\mathrm{TM}}$ to solve Maxwell's equations for a defined set of geometries and materials. For simple geometries the mesh array was reduced to a single unit cell with perfect $\mathrm{E}$ and perfect $\mathrm{H}$ symmetric boundary conditions to reproduce the infinite array as in [12]. Waveports were then placed on the top and bottom to obtain two port S-parameters for light at normal incidence. For more complicated geometries, we used a technique suggested in [13]. Two perfectly matched layers were placed on the top and bottom of the vacuum box to minimize spurious reflections at non-normal incidence in regions that were supposed to simulate free space. Instead of symmetric boundary conditions, pairs of master and slave boundaries were placed to simulate and infinite array and the excitation sources were two plane waves with orthogonal electric field polarizations, see Figure 1.
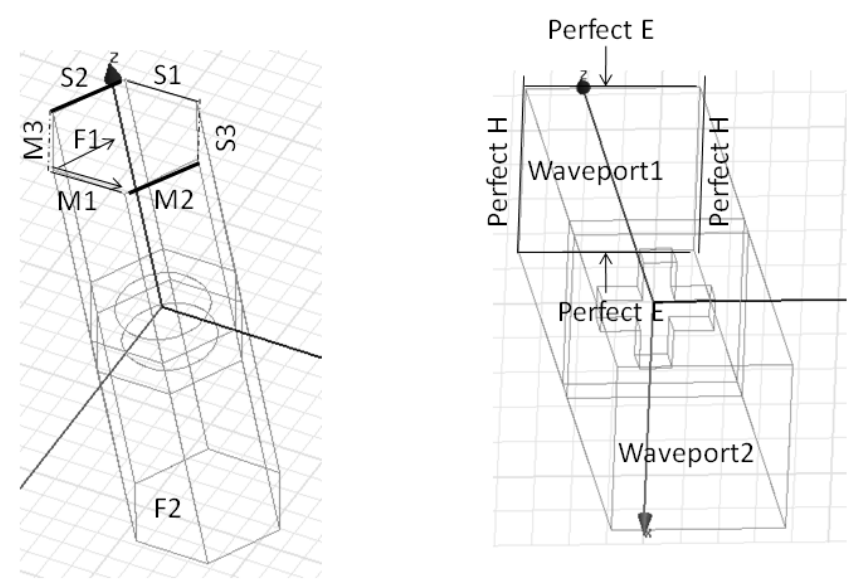

Figure 1. HFSS modeling structures for the cross with perfect $\mathrm{H}$ and perfect $\mathrm{E}$ boundary conditions and the hexagonally close packed filter with master boundaries M1, M2, and M3, slave boundaries S1, S2, and S3, and Floquet ports, F1 and F2. The radiation propagates in the z direction.

We created a hexagonal unit cell, with three sets of master and slave boundary pairs, to pack the aperture opens as closely as possible in order to obtain high resolution of the optical response in the mid - far IR wavelength range. We then defined Floquet ports above and below the cell in terms of vectors oriented along the path of the lattice. This allowed us to define the polarization of the excitation source and analyze the effects of off-normal incidence. Furthermore, we addressed the dependence of the optical properties of the metal on filter performance. The material properties of the bulk metal in the filter were 
represented by a surface impedance approximation in the model. A bulk resistivity for the metal layer of 1.4e-6ohm-cm was used in the simulated results shown in figure 5. The dominant observed difference between this simple treatment and the Drude dielectric function derived from the data in [14] withing the filter passband is the magnitude of the computed absorption. It is increased by $~ 30 \%$ in the latter approximation for a simple reflective surface at normal incidence. For our present purposes this level of accuracy is sufficient. In order to minimize computing resources, we modeled the filter material thickness to be a few penetration depths, which provided reliable numerical results.

In terms of the design of the metal mesh filters, Porterfield et al [12] determined that the performance of a cross-shaped aperture, shown in figure 2, is determined by the periodicity, cross-member length, and cross-member width. The filter profile can be scaled linearly by scaling the dimensions provided that the periodicity is smaller than the wavelength. In [15], Chase et al define the dimensions of the structure differently with the dimension $b$ equal to half the cross-member width and the dimension $a$ equal to half the difference of the periodicity and cross-member length. It was determined that the resonant wavelength is approximately 2.1 times the cross-member length independent of $a$ and $b$ if the ratio of $b / a$ $\leq 1$. Furthermore the bandwidth increases as $b / a$ increases; however, coupling between the crosses is also a significant factor in determining the bandwidth of the filter. Other aspects that influence the shape factor and characteristic of the filter include thickness of the metal as demonstrated in Figure 3 in Ref. 15, the stacking or cascading of filters as demonstrated in [7,17], and the use of other shaped apertures $[7,13,17]$. We then created cross-shaped apertures with cross-member length ranging from $10 \mu \mathrm{m}$ to $50 \mu \mathrm{m}$ to target wavelengths of $20 \mu \mathrm{m}$ to $100 \mu \mathrm{m}$. We also designed cross-shaped apertures with varying $b / a$ ratios to explore bandwidth as was done in [13]. Figure 2 shows the cross dimensions. The parameters in the figure are related to $a$ and b via, $J=2 b$ and $2 a=G-K$. Finally, we designed other shaped apertures, which include squares arranged in a square lattice and circles arranged in both square and triangular lattices, to explore the frequency response of these filters. To ensure that the transmission spectrum was appropriately captured in the model, the frequency sampling was decreased from 10 to 2 $\mathrm{GHz}$ and the response was not noted to change.

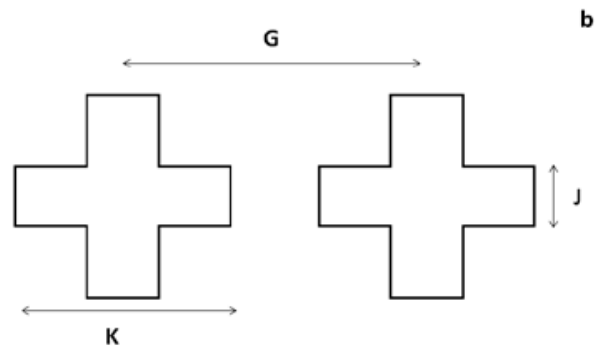

Figure 2. The dimension parameters of the cross shaped filter.

\section{Section II: Fabrication}




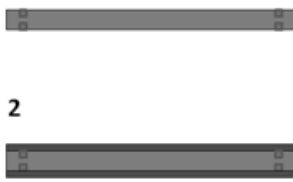

3

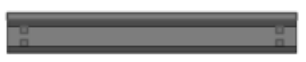

4

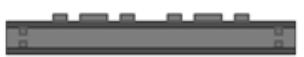

5

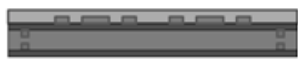

6

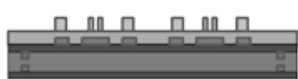

7

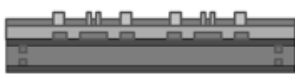

8

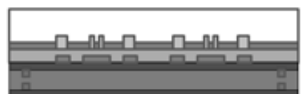

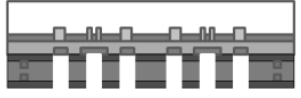

10

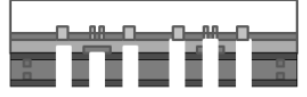

11

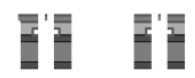

Figure 3. A cross section schematic diagram of the fabrication flow. (1) Pattern front and back alignment marks is Si. (2) Thermally deposit $\mathrm{SiO}_{2}$. (3) Coat Wafer with Parylene sacrificial layer. (4) Pattern and etch Parylene. (5) Deposit Ti/Cu electroplating seed layer. (6) Pattern and etch electroplating mold. (7) Electroplate metal features. (8) Bond wafer to Pyrex wafer. (9) Pattern and etch (DRIE) backetch cut; etch through oxide and exposed seed layer metal. (10) Etch away sacrificial layer and exposed seed layer metal. (11) Release parts.

Our process, depicted in Figure 3, results in a free standing copper resonant metal mesh filter suspended from a silicon support frame. This process can accommodate multiple frame designs for different applications which can also result in a high fabrication throughput for different array architectures. Some examples of different array architectures of realized filters are shown in Figure 4. Our fabrication process can also be extended to make relevant structures, for instance, silicon baffles which can reduce crosstalk between filter elements. In using MEMS fabrication techniques we can achieve one micron alignment tolerance, which is orders of magnitude better than high throughput manual alignment.
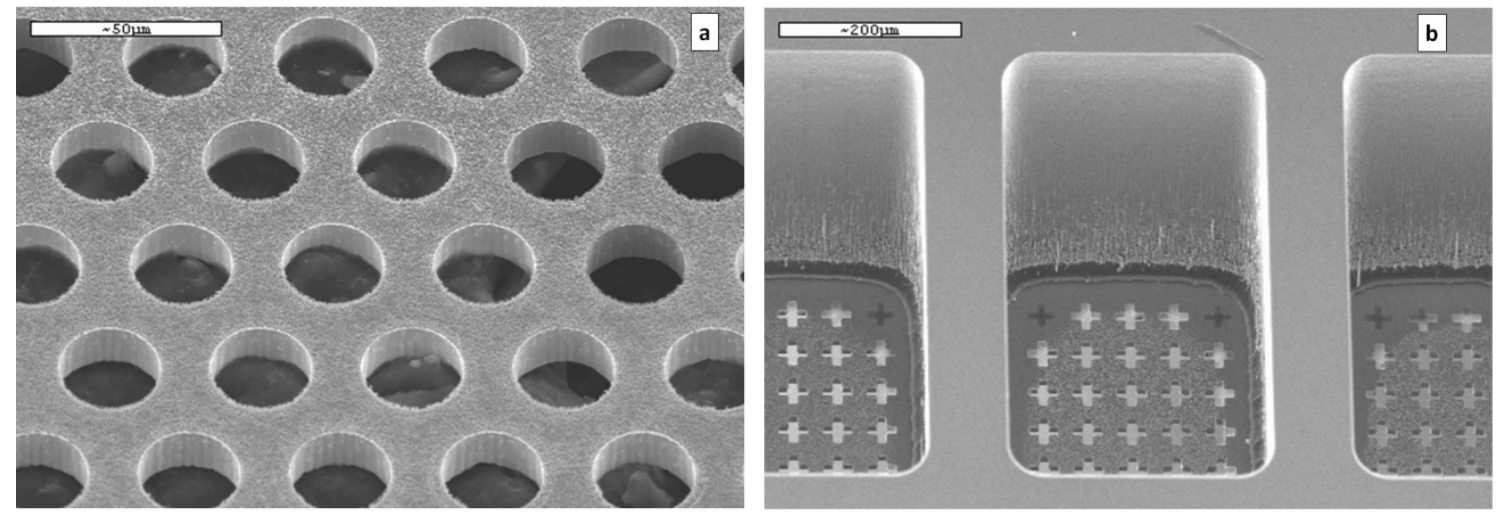

Figure 4. (a) A scanning electron microscope (SEM) image of an electroplated copper mesh filter. The filter consists of a copper sheet with hexagonally close packed circular holes. (b) A SEM image of a linear filter array, in which each filter has cross shaped holes. The dimensions of one aperture are 240 microns x 480 microns. Also visible in this image is the 400 microns thick silicon frame region which 
mechanically supports the filters. The apparent gaps between the silicon and copper are comprised of Parylene-C.

We started the process by depositing a thermal oxide layer on both sides of 4" $\mathrm{Si}(001)$ wafers (resistivity $=10-20 \mathrm{Ohm} \mathrm{cm}$ ) using a tube furnace. The oxide serves as both an insulator to prevent electroplating metal on the back of the wafer and it is an etch stop for subsequent deep reactive ion etching. Front and back alignment marks are then patterned with a positive photoresist, $\mathrm{PR}$, and reactive ion etched in a $\mathrm{CF}_{4} / \mathrm{Ar} / \mathrm{CHF}_{3}$ and $\mathrm{SF}_{6} / \mathrm{O}_{2}$ plasmas, in order to etch $\mathrm{SiO}_{2}$ and $\mathrm{Si}$ respectively. A sacrificial layer of commercially available Parylene-C (Specialty Coating Systems ${ }^{\mathrm{TM}}$ ) is deposited on the wafers and then patterned with positive photoresist. The parylene is then etched in an $\mathrm{O}_{2}$ plasma using a parallel plate barrel asher (Axic).

Parylene has several attractive features which make it a good sacrificial layer. It provides mechanical support and is easily removed in an $\mathrm{O}_{2}$, which does not erode the copper mesh. We find that it is also compatible with the sulfuric acid based electroplating solution used to plate copper. This is because it is both chemically inert, i.e., it does not dissolve in the solution, and an insulator, i.e., plating does not occur on Parylene coated surfaces. It also does not swell in aqueous solution; consequently, delamination of copper on Parylene surfaces is not a problem.

Following the patterning of the sacrificial layer, we electron beam deposited, (base pressure $=2 \times 10^{-7}$ Torr), an electroplating seed layer which consisted of $20 \mathrm{~nm}$ layer of Ti, which served as an adhesion layer between copper and Parylene, and then a $200 \mathrm{~nm}$ layer of $\mathrm{Cu}$ at ambient temperature on the front side of the wafers. As an aside, we observed that $\mathrm{Ti} / \mathrm{Cu}$ seed layer results in a low stress electroplated film [18]. The Cu surface was passivated by immersing the wafer in a 1:4000 Benzotriazole:H20 solution. The filter design was then patterned with SU-8 - 10 (Microchem $\left.{ }^{\mathrm{TM}}\right)$ negative photoresist. $\mathrm{Cu}$ was then electroplated in the SU-8 free regions using a sulfuric acid/copper sulfate based electroplating solution (Microfab-SC) and phosphorized copper anodes at ambient temperature. We found that in order have smooth and near vertical Cu sidewalls the SU-8 thickness must exceed the Cu thickness. We have experience in patterning SU-8 with thickness ranging between 2 and 150 microns. For this work, the SU8 thickness was 16 microns, which more than sufficient for the 10 microns of electroplated $\mathrm{Cu}$. The electroplated $\mathrm{Cu}$ was subsequently passivated in a benzotriazole solution.

In order to fabricate the silicon frame we etched selected regions on the wafers on their backsides. This consisted of bonding the front side of the wafers to Pyrex wafers using an acetone soluble 509 (Crytalbond ${ }^{\mathrm{TM}}$ ) wax. We then ashed away the backside Parylene coating in an $\mathrm{O}_{2}$ plasma and removeed the backside oxide layer via reactive ion etching in a $\mathrm{CF}_{4} / \mathrm{Ar} / \mathrm{CHF}_{3}$ plasma. The frame regions were defined using AZ-4620 positive photoresist and etched using an $\mathrm{SF}_{6} / \mathrm{O}_{2}$ etch and $\mathrm{C}_{4} \mathrm{~F}_{8}$ passivation Bosch process in a deep reactive ion etcher (STS). We reactive ion etched the $\mathrm{SiO}_{2}$ etch stop using a $\mathrm{CF}_{4} / \mathrm{Ar} / \mathrm{CHF}_{3}$ plasma, remove the Parylene in a parallel plate barrel asher with an $\mathrm{O}_{2}$ plasma, stripped the $\mathrm{Ti}$ in 1:10 HF: $\mathrm{H}_{2} 0$, and etched the $\mathrm{Cu}$ seed layer in a $1 \mathrm{M}$ nitric acid solution. The remaining AZ-4620 resiste was ashed away. Partial removal of the SU-8 was accomplished ashing with an $\mathrm{O}_{2} / \mathrm{CF}_{4}$ plasma. Finally the individual dice were released in an acetone bath and any remaining SU-8 was released in ultrasonic solvents.

In the event that the $\mathrm{Cu}$ surface was corroded post processing, it was removed in a $6 \%$ acetic acid solution was passivated using a benzotriazole solution, which was found to protect the parts against corrosion after three months of exposure to atmosphere. 


\section{Section III: Transmission Measurement and Discussion}

The transmission spectra were measured using a Bruker IFS 125 Fourier Transform Spectrometer (FTS). This instrument is equipped with a liquid helium cooled bolometer that allowed us to collect data in the spectral range of 50 to $667 \mathrm{~cm}^{-1}(200-15 \mu \mathrm{m})$, with a $0.5 \mathrm{~cm}^{-1}$ resolution. The data were taken in a focused beam configuration by taking the ratio of the filter transmission relative to a reference hole. The low temperature measurements were taken using an Oxford Instruments liquid helium continuous flow optical cryostat mounted to the Bruker FTS. A correction factor of the ratio of the reference hole area to filter area was used to normalize the measured values because of a mismatch between the shape of the reference hole and the filter geometry. The reference hole was a circular aperture with a $1.5 \mathrm{~mm}$ diameter and the filters that were tested consisted of square shaped mesh regions $1 \mathrm{~mm}$ on a side.
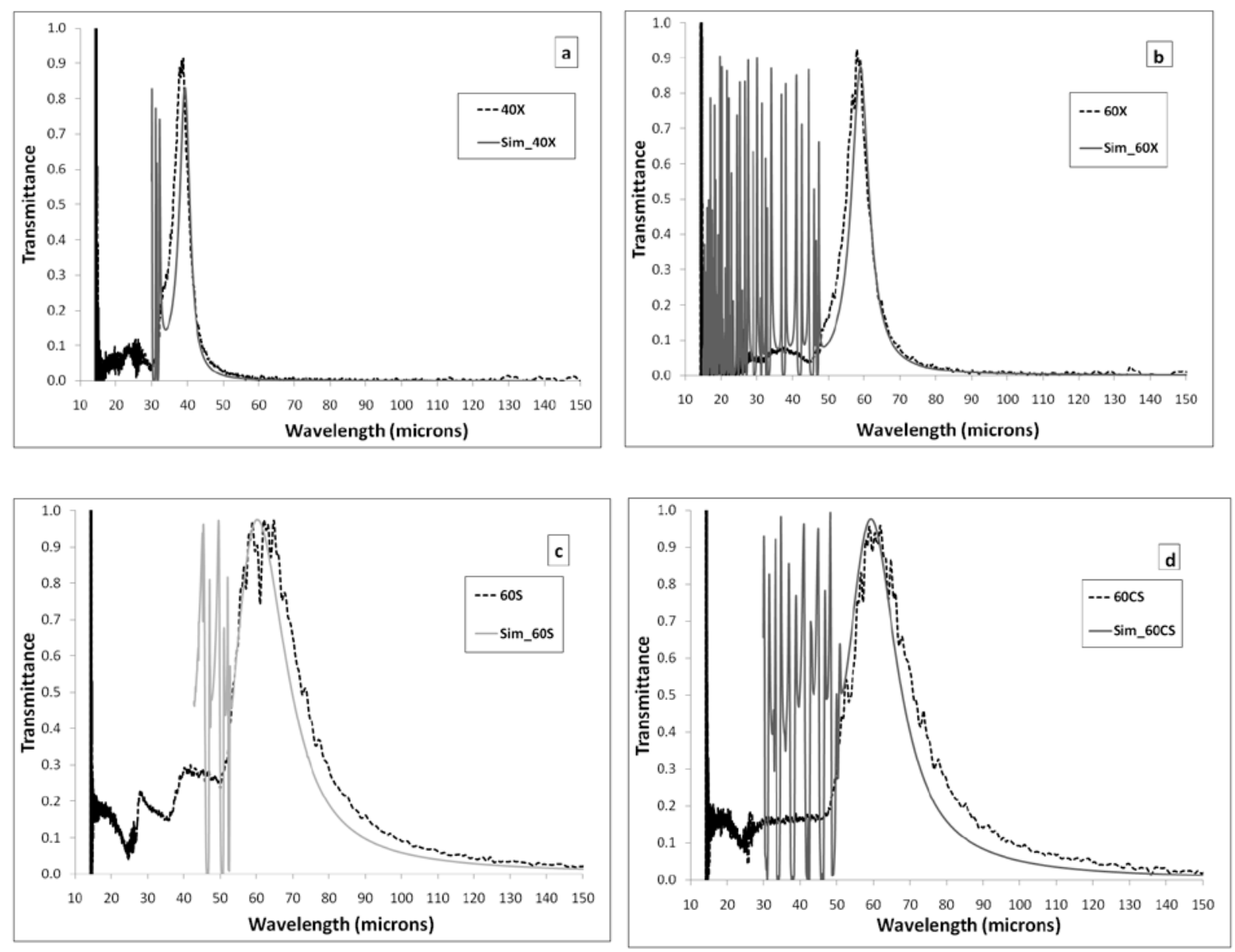

Figure 5. FTS measurement of transmission spectra for a representative sampling of bandpass filters fabricated on a single 4" wafer. Shown in (a) and (b) are filters with cross-shaped holes, indicated by the $\mathrm{X}$, arranged in a square lattice, (c) filters with square holes, indicated by the $\mathrm{S}$, arranged in a square lattice, and (d) circular holes with a hexagonally close packed lattice, indicated by CS. The number in the legend corresponds to the design peak frequency. The hole dimensions are (a) $G=31.5, K=20.5, J=6$ microns (b) $G=47, K=30.5, J=9$ microns (c) $G=52.5, K=34$ microns and (d) $G=58.5, K=39$ microns. The modeled transmission resonances on the left hand side of the plots correspond to expected diffractive 
effect for these structures. These resonances are smeared, because of limited resolution of the FTS.

The passbands are targeted by varying the mesh hole geometry of the filter as discussed above. This facilitates making many filters of different pass bands in a single fabrication run. For instance we yielded 19 dice, each with different filter geometry, on a single 4" Si wafer. The resonance peaks of the passband are located close to the 20,40, and 60 micron design targets and the transmittance at peak frequency for filters having cross, square, and circle shaped holes is between 0.8 and 0.9 at $300 \mathrm{~K}$ as shown in Figure 5 . We note that the agreement between the modeled and measured transmittance is very good. Furthermore the cross patterns give significantly narrower passbands and better long wavelength attenuation than the square or circle patterns. Upon cooling the filters to $7 \mathrm{~K}$, we see, in Figure 6, that the peak transmission for the 60 micron cross shaped filter increases and returns the previously held $300 \mathrm{~K}$ value. Thus, these filters are robust against cryogenic thermal cycling, which is a critical requirement for components designed to operate in the cold environment around the outer planets.
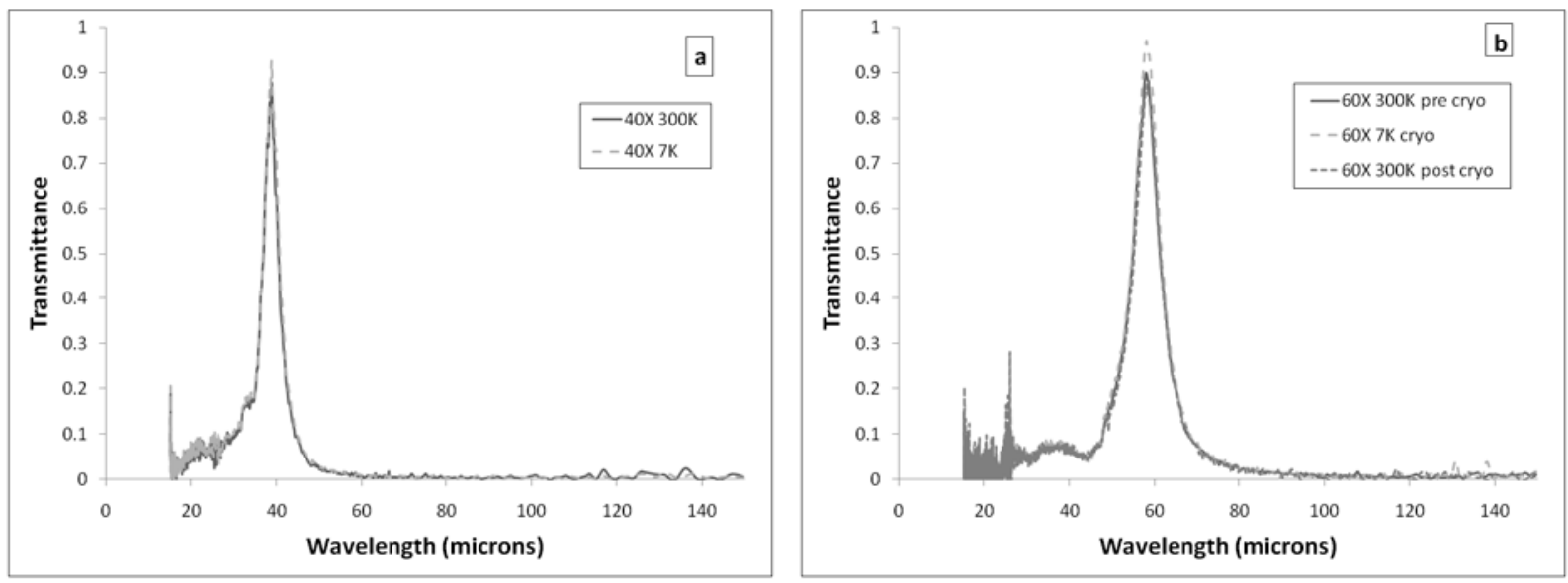

Figure 6. (a) Transmittance spectra of a filter, cross shaped features, designed to have a bandpass at 40 microns at 7 and $300 \mathrm{~K}$. (b) Transmittance spectra of a filter with a 60 micron bandpass design. The spectra were taken at $300 \mathrm{~K}, 7 \mathrm{~K}$, and again at $300 \mathrm{~K}$ in order to ascertain the effects of thermal cycling.

The cross and square shaped mesh hole features exhibit rounding at their corners that was not originally included in our simulations. This rounding occurs because of limitations in defining small feature sizes during photolithographic processing. In order to obtain better agreement with the measured bandpass response, we have performed new simulations in which the meshes have "rounded" hole geometries, as shown in Figure 7. Incorporation of this detail improves the agreement between the observed transmission characteristics and is a perturbation on the initial design. 

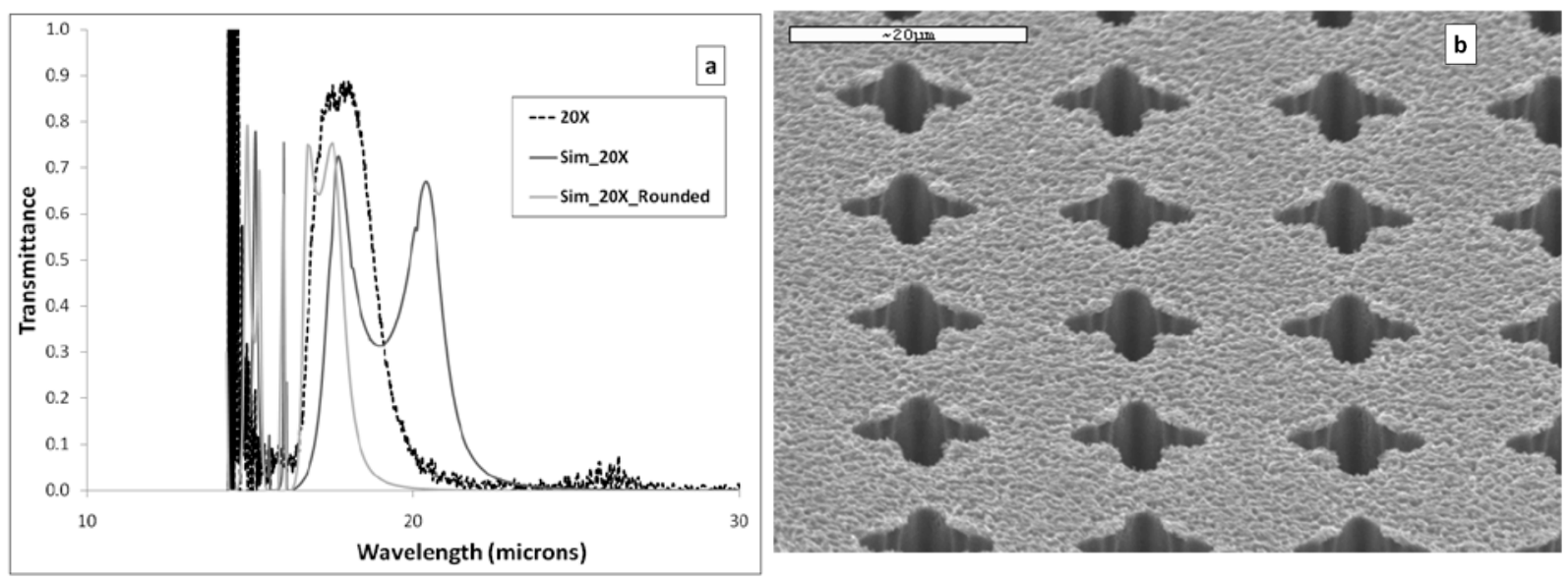

Figure 7. (a) Shows the transmittance for the 20 micron cross shaped filter. The scale has been changed to highlight the passband features. The realized geometry exhibits rounding due to lithographic effects, shown in (b), which caused the filter bandpass response to deviate significantly from the modeled response. The included simulation of the bandpass response with rounded cross features gives much better agreement with measurement.

From both our measured and simulated data, we see that there are limitations in the bandpass response of metal mesh filters. There are both modeled and observed low wavelength resonances as we approach the diffraction limit, i.e., when the free space wavelength $\lambda$ is on the order of any of the characteristic aperture sizes as well as spurious optical response arising from differences in filter design and actual filter geometry as shown in Figure 7. The FTS has much lower resolution below 15 microns, which makes it difficult to resolve low wavelength behavior.
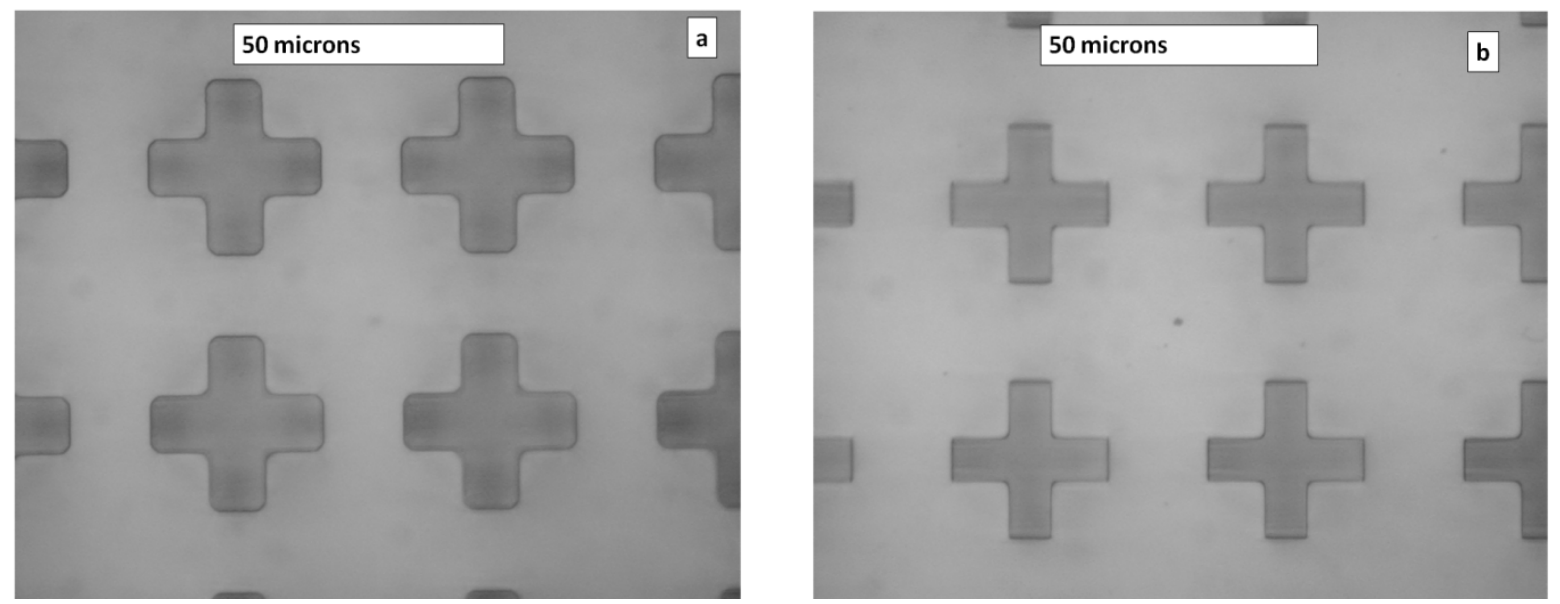

Figure 8. (a) A micrograph of SU-8 posts without using a low pass filter during UV light SU-8 exposure. (b) A picture of the SU-8 posts, in which the same lithography mask was used as in (a) with the inclusion of a SU-8 low pass filter (Omega Optical). The resulting structures are much more precisely defined. 
We can address these issues by improving lithographic capabilities so as to define smaller features. For instance, we found that SU-8 feature resolution can be enhanced by using a low pass filter (Omega Optical) during UV light exposure of the photoresist as shown in Figure 8. This technique can push the feature size resolution to 2 microns. Furthermore, one can use lower throughput and much more costly techniques such as electron beam lithography to define features down to 0.1 micron. Another means to fabricate filters with very small hole dimensions would be to use a self-assembly technique, for instance, by using block copolymers [19] to fabricate an electroplating mask. In all instances however, there is a penalty for using smaller features, namely a reduction in transmittance. Perhaps the most efficient means of eliminating such unwanted resonances is to integrate the metal mesh bandpass filter with an antireflection coated crystal such as $\mathrm{SrF}_{2}$ whose Restrahlen band would reject this spectral region by reflection.

Another limitation of single layer metal mesh filters is their narrow bandwidth. One means of improving bandwidth is filter stacking [6]. Our models predict that stacking the filters will produce a sharper roll off and wider passband but also a lower transmittance as shown in Figure 9. Using our fabrication approach the silicon frame can act as a spacer when stacking.

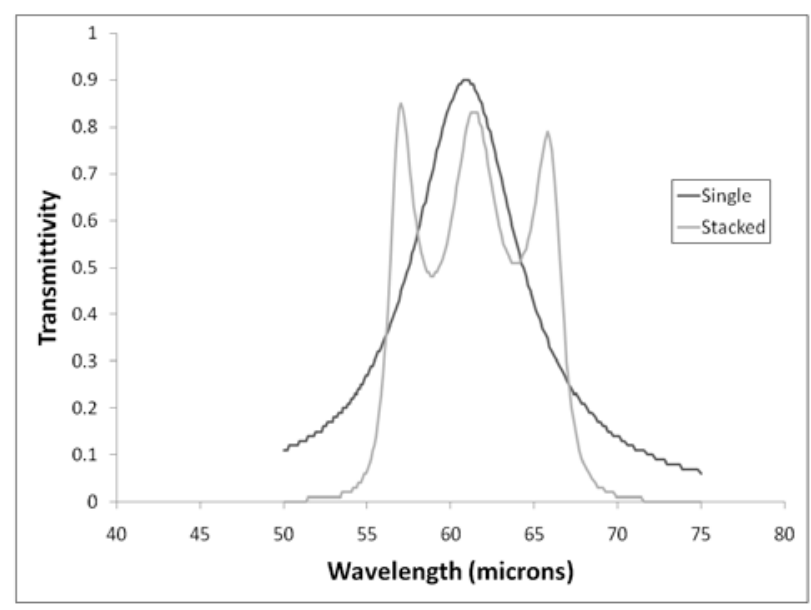

Figure 9. Simulated comparison of bandpass response between using a single filter with cross shaped features and using two filters stacked 100 microns apart. We note the sharper roll off and increased effective full width at half maximum. The cross dimensions are $G=47, K=10, J=32$ microns.

\section{Section IV: Future outlook}

We envision integrating the filter array with a detector array to build a radiometer. The focal plane assembly consists of a thermopile array aligned with a filter array, and baffles separating the respective array elements to prevent detector cross talk and reduce non-normal incident light through the filter. A combination of low and high pass filter windows are placed in front of the filter array in order to remove the extra resonances from out-of-band response. An advantageous feature of the process described in this paper is that the extension to making the baffles from the substrate is straightforward. Likewise, characteristic needs can potentially be satisfied by judicious fabrication choices. Since the baffles need to be good absorbers, for example, possible solutions are to use degenerately doped silicon wafers, or an 
aluminum coating with black anodization. We anticipate being able to satisfy mission requirement with custom designs of filter integration for a wide variety of radiometric instruments.

\section{Section V: Conclusion}

We have succeeding in making a low mass, radiation hard, small volume, free standing metal mesh filters with silicon support frames using common semiconductor fabrication process. The transmissivity is in the $0.8-0.9$ range, and the measured bandpass response is in good agreement with the modeled response. The filter bandpass response contains more than one resonance, which was anticipated in the model. These additional resonances can be eliminated by fabricating much smaller features or by employing a low pass window with an antireflective coating. Our models show that by stacking the filters, we can increase their bandwidth. The novel fabrication process that we present can also be extended to hybridize the filter array with the detector array so as to construct a radiometric instrument.

This work was supported by a directed FY2011 IRAD award through Brook Lakew from the NASA Goddard Space Flight Center. This work was also supported by an appointment to the NASA Postdoctoral Program at the Goddard Space Flight Center, administered by Oak Ridge Associated Universities through a contract with NASA.

\section{References}

[1] NASA Decadal Survey "Visions and Voyages for Planetary Science in the Decade 2013 - 2022”, The National Academies Press (2011).

[2] K. Clark, A. Stankov, R. Pappalardo, M. Blanc, R. Greeley, J. Lebreton, NASA/ESA Europa Jupiter System Mission Joint Summary Report (2009)

[3] J Boldt, “Overview of JEO model payload” EJSM Instrument Workshop July 15-17, 2009. K.B. Clarke, T Magner, C Erd, “Europa Jupiter Systems Mission Overview” EJSM Instrument Workshop July 27-29, 2010

[4] V. P. Tomaselli, D. C. Edewaard, P. Gillan, and K. D. Möller, "Far-infrared bandpass filters from cross-shaped grids," Appl. Opt. 20, 1361-1366 (1981)

[5] H.G. Booker, "Slot aerials and their relation to complementary wire aerials (Babinet's principle), " Proc. IEE(London) Vol. 93, Pt. 3A, pp. 620-626, 1946

[6] Peter A. R. Ade, Giampaolo Pisano, Carole Tucker and Samuel Weaver, "A review of metal mesh filters", Proc. SPIE 6275, 62750U (2006)

[7] G. D. Holah, "Far-infrared and submillimeter-wavelength filters," in Infrared and Millimeter Waves, K. J. Button, ed. (Academic, New York, 1982), Vol. 6, pp. 305-409.

[8] Akgun, U.; Bruecken, P.; Ayan, A.S.; Duru, F.; Olson, J.; Albayrak, E.A.; Gulmez, E.; Mestvirishvili, A.; Onel, Y.; Schmidt, I.; , "Radiation damage and light transmission studies on air core light guides," Nuclear Science, IEEE Transactions on , vol.53, no.3, pp. 1547- 1550, June 2006. G. Wang et al., “Proton Beam Modification of Isotactic Polypropylene.” Nucl. Instrum. Methods B, 27, 410 (1987). 
[9] P. F. Goldsmith, “Quasi-Optical Systems: Gausian Beam Quasioptical Propagation and Applications,” 1998, IEEE Press, NY

[10] P. G. Huggard, M. Meyringer, A. Schilz, K. Goller, and W. Prettl, "Far-infrared bandpass filters from perforated metal screens," Appl. Opt. 33, 39-41 (1994)

[11] Arline M. Melo, Mariano A. Kornberg, Pierre Kaufmann, Maria H. Piazzetta, Emílio C. Bortolucci, Maria B. Zakia, Otto H. Bauer, Albrecht Poglitsch, and Alexandre M. P. Alves da Silva, "Metal mesh resonant filters for terahertz frequencies," Appl. Opt. 47, 6064-6069 (2008)

[12] D. W. Porterfield, J. L. Hesler, R. Densing, E. R. Mueller, T. W. Crowe, and R. M. Weikle II, "Resonant metal-mesh bandpass filters for the far infrared," Appl. Opt. 33, 6046-6052 (1994)

[13] G. Pisano, P. A. R. Ade, and S. Weaver, "Polarization effects investigations in quasi-optical metal grid filters.” Infrared Phys. Technol. 48, 89-100 (2006).

[14] M. A. Ordal, L. L. Long, R. J. Bell, S. E. Bell, R. R. Bell, R. W. Alexander, Jr., and C. A. Ward, "Optical properties of the metals $\mathrm{Al}, \mathrm{Co}, \mathrm{Cu}, \mathrm{Au}, \mathrm{Fe}, \mathrm{Pb}, \mathrm{Ni}, \mathrm{Pd}, \mathrm{Pt}, \mathrm{Ag}, \mathrm{Ti}$, and $\mathrm{W}$ in the infrared and far infrared," Appl. Opt. 22, 1099-1119 (1983)

[15] S. T. Chase and R. D. Joseph, "Resonant array bandpass filters for the far infrared,” Appl. Opt. 22, 1775-1779 (1983)

[16] V. V. Kubarev et al., "Development of metal mesh based quasi-optical selective components and their application in high-power experiments at Novosibirsk terahertz FEL.” Proceedings of FEL, 2007, pp. 89-92.

[17] Carsten Winnewisser, Frank Lewen, Jochen Weinzierl, and Hanspeter Helm, "Transmission Features of Frequency-Selective Components in the Far Infrared Determined by Terahertz Time-Domain Spectroscopy," Appl. Opt. 38, 3961-3967 (1999)

[18] We also tried $\mathrm{Ti} / \mathrm{Au}, \mathrm{MO}_{2} \mathrm{~N}$, and TiN as seed layers. For $\mathrm{MO}_{2} \mathrm{~N}$ and TiN the electroplated film had poor adhesion to the substrate and $\mathrm{Ti} / \mathrm{Au}$ had high surface roughness.

[19] C. Tang, E. M. Lennon, G. H. Fredrickson, E. J. Kramer, C. J. Hawker, "Evolution of Block Copolymer Lithography to Highly Ordered Square Arrays.” Science, 2008, Vol. 322, No. 5900, pp. 429432. 